\title{
SOURCES OF INTERPLANETARY SHOCKS
}

\author{
J. G. Luhmann \\ Institute of Geophysics and Planetary Physics, University of California, \\ Los Angeles, CA 90024-1567, U.S.A.
}

\begin{abstract}
The sources of interplanetary shocks are reviewed from the standpoint of today's knowledge. Recent progress from both new and accumulated observations and models indicates that contributions from the two major sources of the shocks: CMEs and stream interactions, have distinctive radial, latitudinal and temporal dependencies. The need for global statistical surveys over several solar cycles, and models that take into account the complexity of the solar wind, is apparent from this current perspective.

\section{INTRODUCTION}

Interplanetary shocks are of interest because of their role in both particle acceleration and geomagnetic activity, as well as for the basic plasma physical insights that they provide. The two primary sources of interplanetary shocks, coronal transients known as coronal mass ejections (CMEs) and fast/slow solar wind stream interactions, have been recognized since the early years of in-situ interplanetary medium measurements $/ 1,2 /$. Recently, longer term, broader ranging observations and new modeling efforts have revealed details of the spatial and temporal attributes of both the sources and the shocks that they produce. In particular, Ulysses observations at high heliolatitudes, Voyager and Pioneer observations at large heliocentric distances and over several solar cycles, and 3-D numerical solar wind stream interaction models of Pizzo have provided new insights. This review describes some of these new results in the context of some earlier observational and theoretical work. As a result of these progressing efforts, we now better appreciate how the contributions of the two sources should vary as a function of radial distance from the Sun, latitude from the heliographic equator, and phase of the solar cycle. However, observational analyses of interplanetary shocks over the full range of heliospheric locations and solar activity conditions have yet to be pieced together to test our expectations.
\end{abstract}

\section{SOURCE CONTRIBUTIONS AS A FUNCTION OF SOLAR CYCLE PHASE}

The coronal transient sources of interplanetary shocks can be accounted for almost wholly by CMEs. CMEs are defined as transient changes in the corona that are often observed in coronagraphs as expanding ejected loops of material of enhanced density [e.g., /3/]. As illustrated by Figure 1 from Gosling /4/, the CMEs drive interplanetary shocks if they move or expand at super-magnetosonic velocities with respect to the ambient solar wind. Several studies have now been carried out [e.g., 15, 6/] that demonstrate the close correlation between the rate at which fast coronal mass ejections are emitted from the lower corona and the rate of occurrence of interplanetary shocks observed within $1 \mathrm{AU}$ of the Sun. Some of these data are reproduced in Figure 2, which also shows the strong association between the interplanetary shock occurrence near $1 \mathrm{AU}$ and sunspot number, the traditional solar activity indicator $17,8 \%$.

The relationship between CMEs and solar flares has been clarified [e.g., /9/], but the physics that determines the CME velocity at its origin remains unknown. Mach numbers of CME-driven shocks are typically low, with values $\sim 2 / 10 \%$. The shocks are generally followed by counterparts of planetary magnetosheaths in which the interplanetary magnetic field (IMF) is compressed and reoriented, and the 

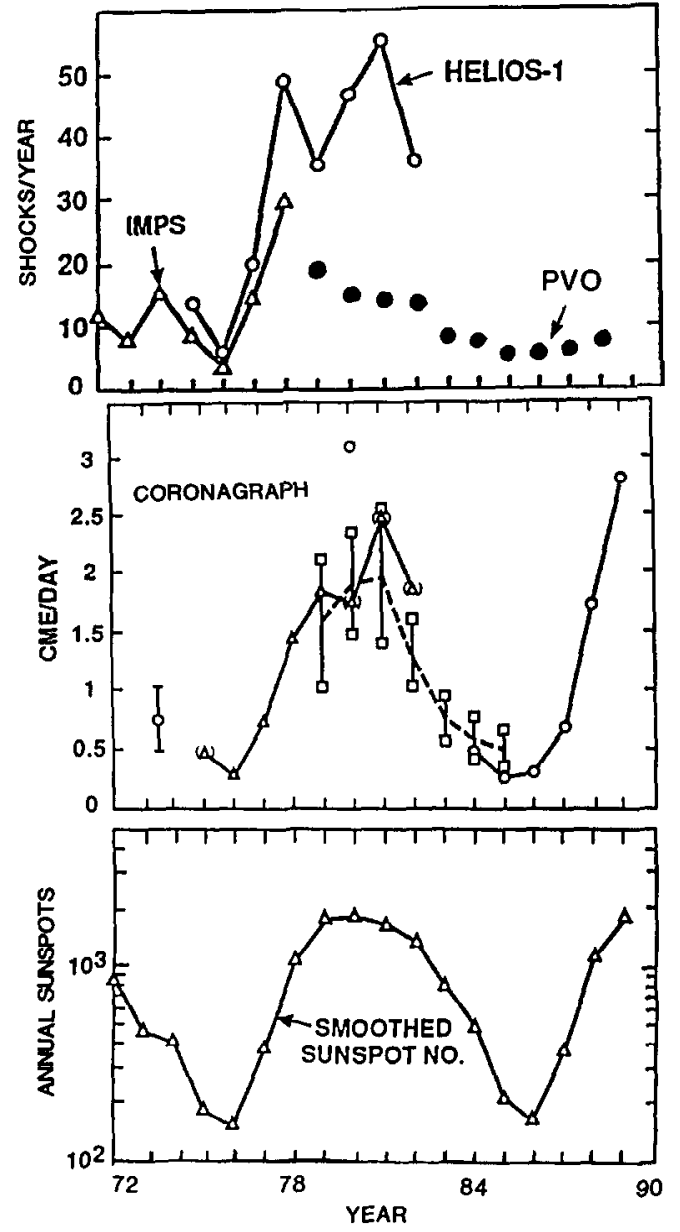

Fig. 1. Illustration of a fast coronal mass ejection's effects on the interplanetary medium (from Gosling /4/).

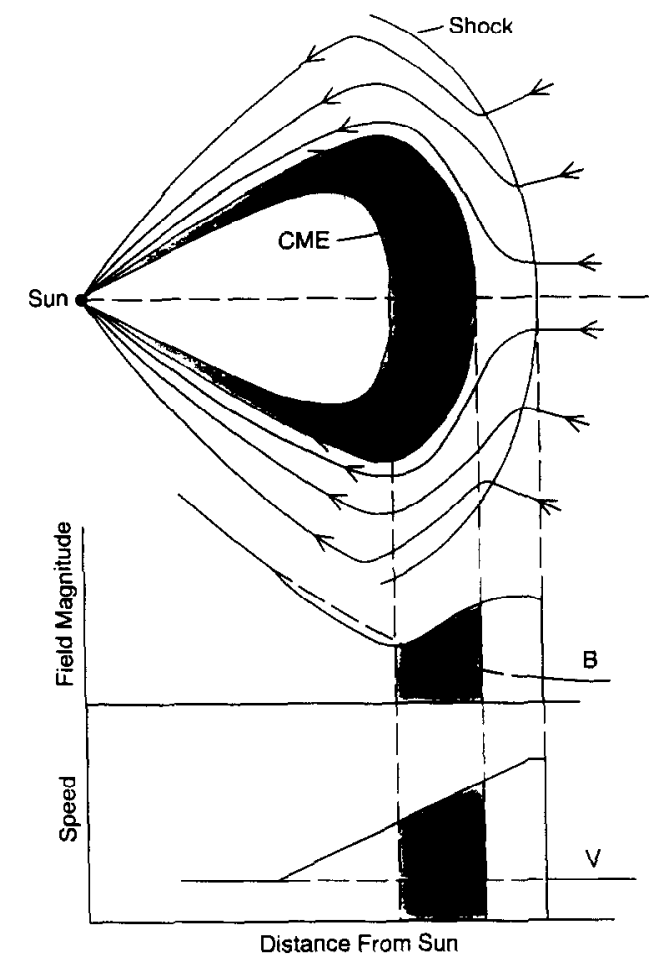

Fig. 2. (Adapted from Webb and Howard 16/.) The rate of interplanetary shock occurrence between 1972 and 1988 (top), including the $0.7 \mathrm{AU}$ Pioneer 12 (PVO) data of Lindsay et al. 15/; the rate of CMEs observed by coronagraphs (center); the smoothed annual sunspot number (bottom). In the top panel the rates have been "normalized" for sampling but by different methods.

solar wind density enhanced, by virtue of the obstacle relative motion $/ 11 \%$ In about $30 \%$ of the cases observed, the "body" of the detected CME in interplanetary space has the magnetic field signature of a flux rope [e.g., /12, 13/] (also see Figure 1). However, CMEs are more generally identifiable as enhanced, smoothly varying magnetic field structures wherein the electron heat flux exhibits bidirectionality suggesting connection to the Sun $/ 14 /$. Occasionally transient interplanetary shocks without a following CME body are detected $15,12 /$, but these cases are a minority and can be attributed to glancing encounters with the CME "sheath" since CMEs typically have a finite heliolongitude extent $\leq 100^{\circ} / 15 \%$. It is expected from intuitive arguments and simple models that the momentum (or energy) of the ejected mass determines the radial range over which the CME remains fast enough to produce a preceding shock [e.g., /16/]. Since both momentum exchange with the ambient solar wind and shock dissipation processes occur as they travel outward, only the largest and fastest CMEs are expected to reach the outer solar system. Hundhausen et al. $/ 17 /$ have recently shown that the speed distributions of CMEs observed with coronagraphs do not show a marked solar cycle dependence. One would thus expect roughly the same solar cycle signature in the frequency of CME-driven interplanetary shocks in both the inner and outer heliosphere. 


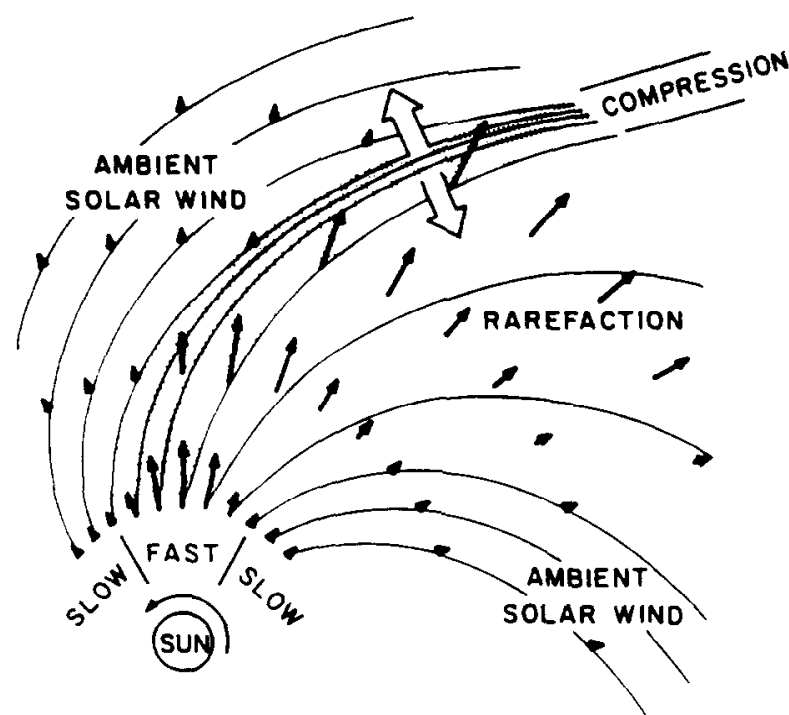

Fig. 3. Illustration of the formation of a stream interaction region (from Pizzo /20/).
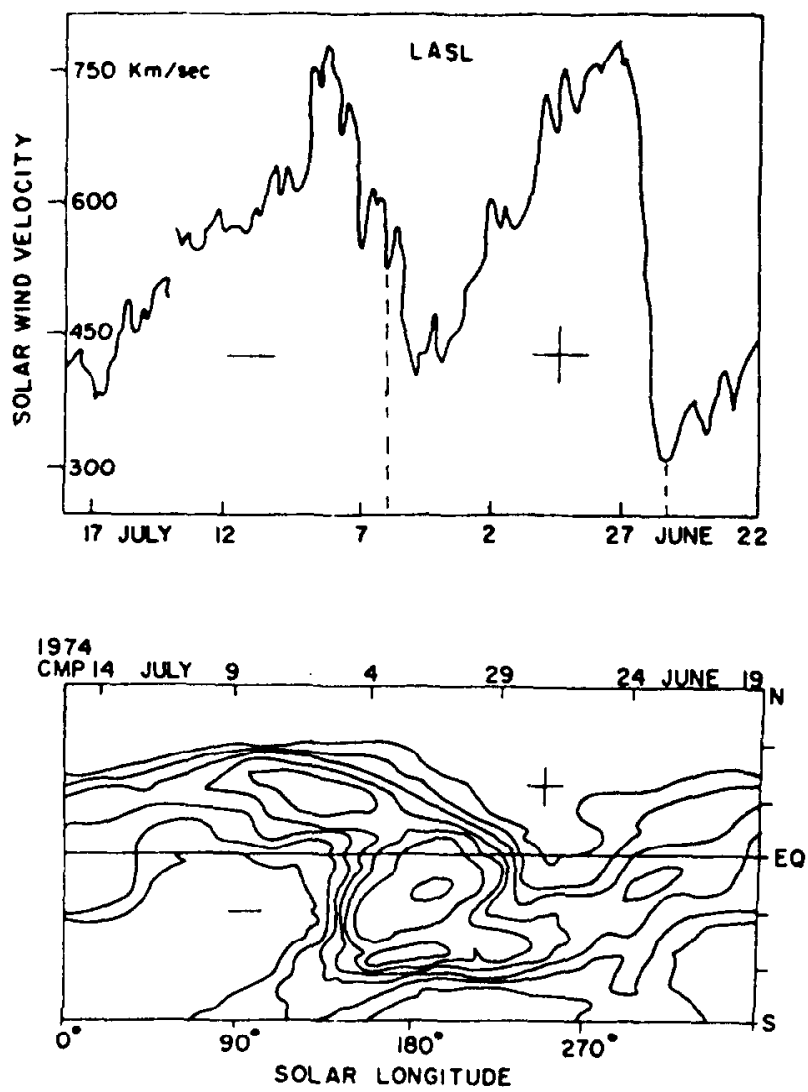

MAUNA LOA $K$ CORONAL OBSERVATIONS 1.5 RO

Fig. 4. Illustration of the correspondence between polar coronal holes as seen in K-coronometer data and high speed solar wind streams (from Zirker 122/).
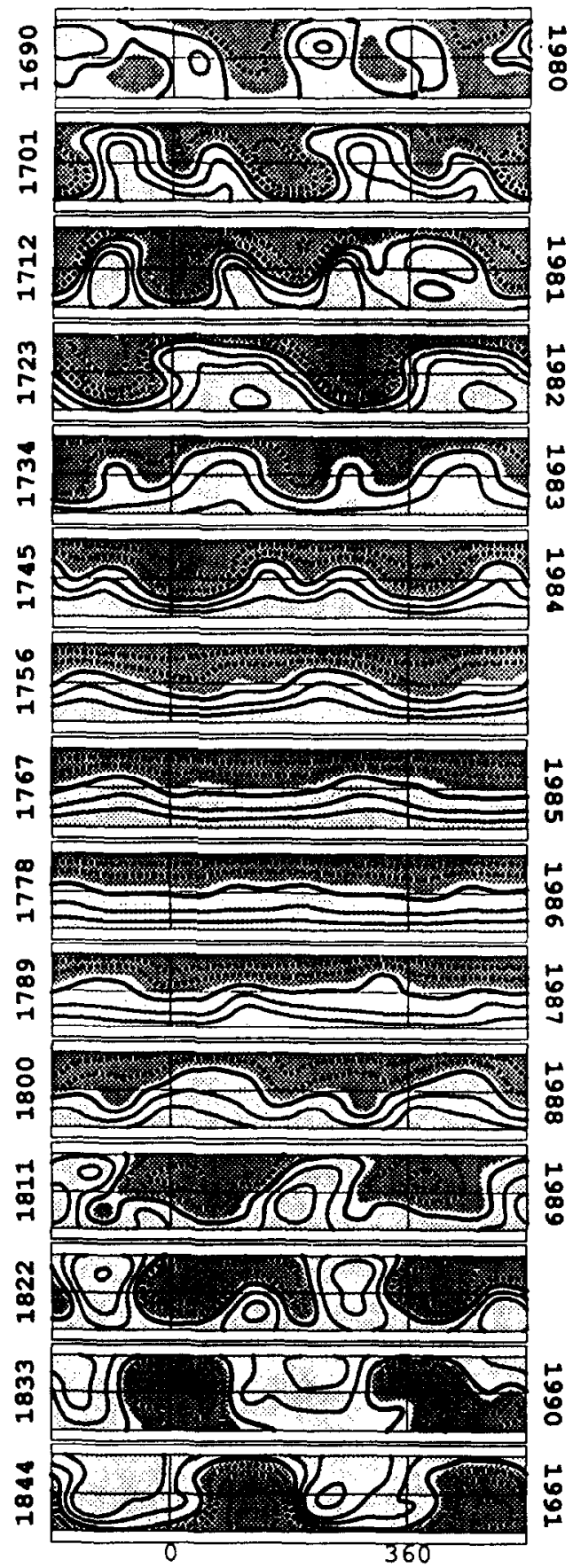

Fig. 5. Solar cycle evolution of the neutral sheet on the solar wind source surface (from Hoeksema /23/). 


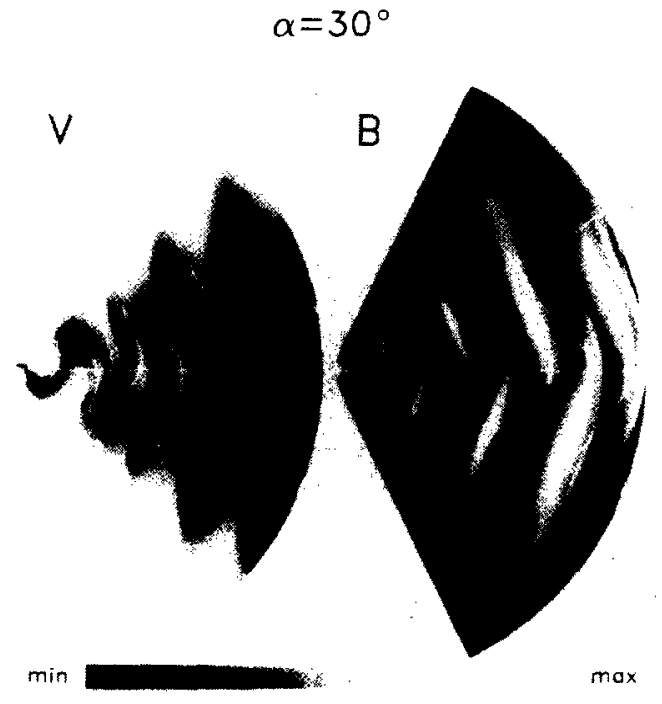

$$
\alpha=30^{\circ}
$$

Fig. 6. Longitudinal cuts through the 3-D MHD stream interaction simulation of Pizzo $/ 19 /$, showing velocity and magnetic field magnitude contours for a dipolar (planar) neutral line tilted $30^{\circ}$ from the solar rotation axis. The white line shows the neutral sheet location.
PVO Disturbance and Sunspot Number Comparison

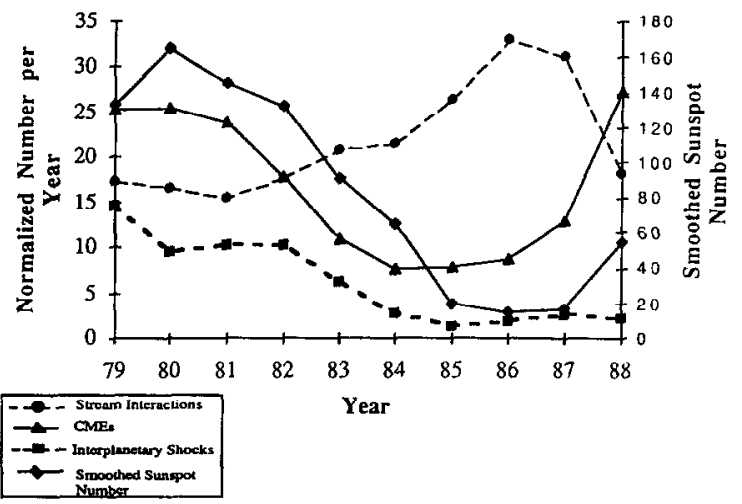

Fig. 7. Solar cycle dependence of CME, stream interaction and shock occurrence over the previous cycle as determined from $0.7 \mathrm{AU}$ data by Lindsay et al. $15 \%$.

\section{SOURCE CONTRIBUTIONS AS A FUNCTION OF RADIAL DISTANCE}

Earlier studies of Helios data obtained between 0.3 and $1.0 \mathrm{AU}$ (in particular, those of Volkmer and Neubauer /24/ and Sheeley et al. 125/), coupled with Pioneer and Voyager spacecraft studies (by Mihalov /26/, and Gazis and Lazarus /27/ for example) out to $~ 20 \mathrm{AU}$, provided the first indications of the radial evolution in interplanetary shock numbers and strength. From these results it is apparent that within $\sim 2 \mathrm{AU}$ almost all interplanetary shocks originate from CMEs, while corotating shocks from stream interactions contribute heavily to what is observed between $\sim 3$ and 10 AU. The few shocks observed at still larger distances appear to be complex in that they appear to have formed from merged stream interaction regions or merged transient and corotating structures [e.g., /28/].

Most statistical studies have either focused on shocks with a clear connection to CMEs or solar activity [e.g., /25, 26/], or presented results without attempting to distinguish between coronal transient and stream sources [e.g., /27/]. Figure 8 , which is drawn from a variety of published results, shows how both the numbers of shocks and their strengths depended on heliocentric distance in a number of different studies. When considering these displays, one must remember that the different data sets were analyzed using different criteria for both shock identification and averaging. Some of the data are averages while others are for single shock observations. Any dependence on heliolatitude, which must occur given the nature of the sources, is neglected.

Figure 8a shows rates of occurrence versus radial distance. Because the occurrence rates are a function of both phase of the solar cycle and details such as the configuration and inclination of the heliospheric neutral sheet, no radial distribution should be used as a model except in the broadest terms. For this set of results a practically constant rate of $\sim 0.05$ transient shocks per day is indicated out to at least $\sim 1$ 


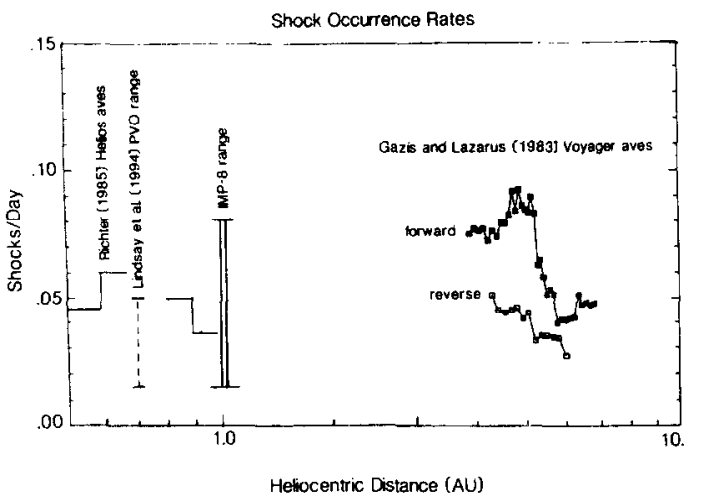

Fig. 8a. Shock occurrence rates at different heliocentric distances. The 1 AU range is derived from the IMP-8 data in Figure 1 and spans the 1975 solar minimum.to the middle of the rising phase of activity. The $0.7 \mathrm{AU}$ range spans the 1979-80 solar maximum through the 1986 minimum. Helios averages are from Richter et al. 133/ for 1975-80, and the Voyager averages for both forward and reverse shocks during 1977-80 come from the study of Gazis and Lazarus /27/.

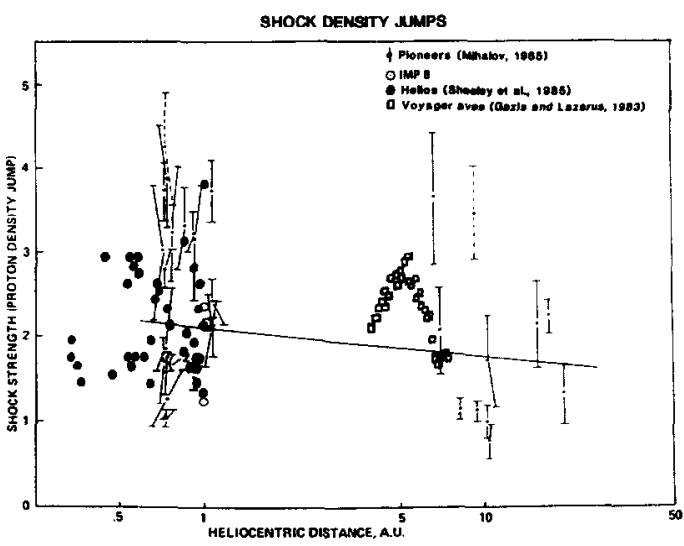

Fig. $8 \mathrm{~b}$. Shock strengths as seen in the density jumps at different heliocentric distances. The individual shock observations are from Sheeley et al. 's (25/ study of 1979-82 Helios data and from Mihalov's /26/ study of 1978-80 Pioneer (and IMP-8) data. Voyager 1977-80 forward shock averages from Gazis and Lazarus /27/ are also shown.

AU. The rate of more distant shocks, which includes corotating types, grows with radial distance as might be expected, peaking at $\sim 0.10$ per day at $\sim 5 \mathrm{AU}$. In a period of generally weak solar activity the transient rates would be much lower (as discussed in the previous section). Likewise, for a period associated with a flat equatorial neutral sheet, the rate of stream-related shocks at $>2 \mathrm{AU}$ distances would be less, or higher inclination neutral sheets during this period would produce larger corotating shock rates and/or higher shock strengths that peak at larger distances than $5 \mathrm{AU}$. The radial profile of interplanetary shock occurrence may be expected to be different for each solar cycle, and the two parts of the profile, from CMEs and stream interactions, will vary independently as do their sources. The only "predictables", as far as source rate variations are concerned, are the positive correlation of CME occurrence and solar activity, and the potential prevalence of stream-related shocks during periods of moderate to low activity. As a consequence, interplanetary shocks from all sources should show a clear solar activity cycle at radial distances $\leq 2 \mathrm{AU}$ but a different cycle at larger distances depending on the evolution of the stream structure. The distinction between the two sources at these larger distances is expected to be difficult considering that the CMEs propagate into and through the stream interaction regions with their own shocks.

In Figures $8 b$ and $8 c$ two indicators of shock strength, density and velocity jumps, are shown as a function of distance. The density jumps in Figure 8a for the "transient" cases of Mihalov /26/ appear to show a weakening trend with increasing distance. Moreover, for the same period fewer transient events are observed beyond $10 \mathrm{AU}$ than are observed at and inside $1 \mathrm{AU}$, as if attrition due to deceleration and dissipation has taken its toll. However, the averages of Gazis and Lazarus /27/, which may be primarily from stream-interaction shocks, peak at about $5 \mathrm{AU}$ as if the shocks strengthened with distance up to that radius. The velocity jumps in Figure $8 \mathrm{c}$ indicate a deceleration in the Helios medians, but a diversity of trends otherwise. For example, the possible corotating shocks from Voyager seem to accelerate and then decelerate, while the transient shocks of Mihalov show only a hint of a deceleration trend. In general Figure $8(\mathrm{a}-\mathrm{c})$ suffers from the lack of a standard of treatment in the study of interplanetary shock statistics, but it is thereby a true representation of our state of observational knowledge. 


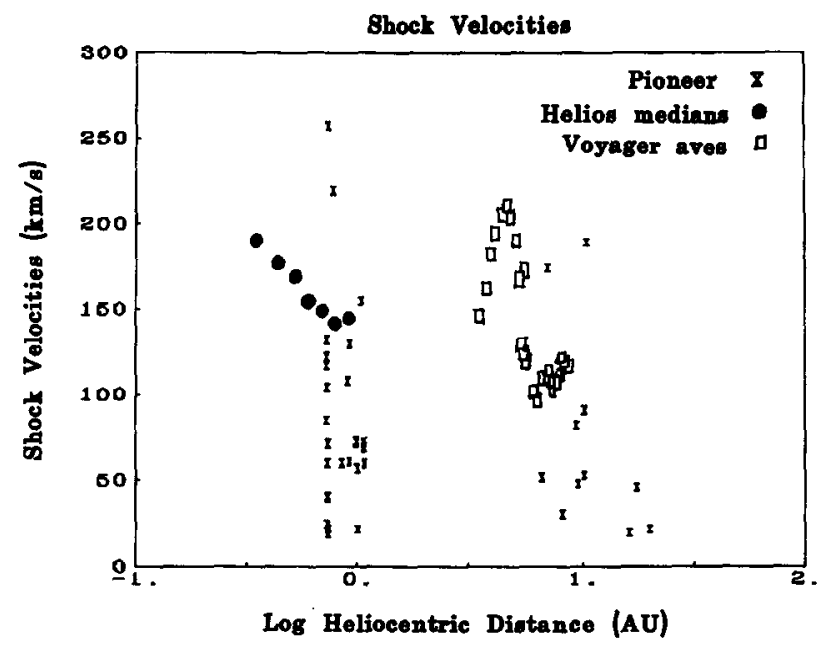

Fig. 8c. Shock strengths as seen in the velocity jumps at different heliocentric distances. The individual shock observations are from the same Mihalov /26/ study as in Figure 2b. Helios medians were derived from plots in Volkmer and Neubauer /24/. The Voyager averages are from the Gazis and Lazarus study $127 /$.

One additional factor that should be mentioned in connection with shock strength evolution is the effect of increasing solar wind magnetosonic Mach number with radial distance $/ 29 /$. As a disturbance moves outward, the relative obstacle speed required to produce a leading shock diminishes (from $\sim 400 \mathrm{~km} \mathrm{~s}$ at $0.1 \mathrm{AU}$ to $\sim 40 \mathrm{~km} \mathrm{~s}^{-1}$ at $30 \mathrm{AU}$ ). Those shocks that reach the outer heliosphere will therefore have a prolonged lifetime as the strengthening effect of decreasing Mach number competes against their dissipation and deceleration. In actuality, few interplanetary shocks are observed beyond $\sim 20 \mathrm{AU} / 28 /$. Many do not seem to persist to even $10 \mathrm{AU} / 26 /$ as momentum transfer to the ambient medium, dissipation processes, and intermixing of the plasmas erase the signatures of the different solar sources. Interplanetary shocks are thus primarily a phenomenon of the inner and middle heliosphere.

\section{SOURCE CONTRIBUTIONS AS A FUNCTION OF HELIOLATITUDE}

The Ulysses mission provided the first opportunity to study in some detail the evolution of the streaminteraction structures as a function of heliolatitude. This mission started its out-of-ecliptic phase in early 1992, when the heliospheric current sheet was warped and well-developed coronal holes were present. Gosling et al. $130 /$ found that as the spacecraft moved poleward, reverse interplanetary shocks associated with the stream interaction regions persisted to higher heliolatitudes than the forward shocks. These observations can be understood in terms of the models of Pizzo /19/ which show how flow deflections from the tilted stream fronts can drive the associated reverse shocks away from the ecliptic while the forward shocks travel in the opposite direction. This behavior is a consequence of the solar wind speed heliolatitude gradient. It should be appreciated that although the neutral sheet in the heliosphere is generally not coincident with the stream interfaces [e.g., /31/], its inclination near the Sun determines the pattern of velocity shear to large distances.

The Ulysses mission results have also shown that CMEs are present at moderate to high heliolatitudes and that they can cause shocks there in spite of the high ambient speed $\left(\sim 800 \mathrm{~km} \mathrm{~s}^{-1}\right)$ of the polar solar wind if their speed of expansion is supermagnetosonic. In this case both forward and reverse shocks are seen in conjunction with the CME $/ 32 /$. These observations raise some remaining issues. In particular, we do not fully appreciate the importance of expansion versus mass motion of a CME in producing shocks. Moreover, as mentioned earlier, CMEs move through the background of the sometimes very complex stream structure depicted by Pizzo/19/ rather than through a uniform ambient solar wind. They should undergo complicated distortions as a result, and their associated shocks will mirror these distortions. It may also happen that a CME is moving supermagnetosonically with respect to the ambient medium over only part of its interaction front (e.g., its low latitude portion). CMEs launched into realistic models of stream structure such as Pizzo's /19/ can in principle shed light on the consequences of a nonuniform solar wind for the resulting interplanetary shocks. 
Stream interaction-related shocks occur when the longitudinal velocity shear between two adjacent solar wind streams is sufficiently large [e.g., /18, 19/]. They are caused by the compression region that forms between the streams as illustrated in Figure 3 (from $/ 20 /$ ). The compression region appears as an obstacle to the slower upstream flow and a barrier to the faster downstream flow, thereby producing a forward upstream and reverse downstream shock pair. The spatial extents and lifetimes of stream interaction shocks depend on the configuration and solar cycle evolution of the magnetic neutral line at the source surface of the solar wind. The solar wind velocity at the source is organized with respect to the neutral line such that it is at a minimum of $\sim 300 \mathrm{~km} \mathrm{~s}^{-1}$ at the neutral line and increases to $\sim 800 \mathrm{~km} \mathrm{~s}^{-1}$ at angular distances from the neutral line of $\sim 15^{\circ} / 21 /$. As illustrated by Figure 4 (from $/ 22 /$ ), the neutral line configuration determines where the high speed polar coronal hole flows protrude into the solar equatorial region where solar rotation wraps them into the characteristic Archimedean spiral stream structure. Its rate of evolution determines the persistence or perceived recurrence of the stream interaction-related shocks as they corotate with the Sun together with the stream structure.

The location of the magnetic neutral line on the source surface has now been determined for almost two solar cycles $/ 23 /$. Some examples of its appearance at different phases are given in Figure 5. It appears that the dipolar contribution to the Sun's magnetic field undergoes a rotation from pole to equator as solar activity increases from minimum to maximum levels. Superposed on the associated planar neutral line as it changes from an equatorial to polar inclination are warps from quadrupole and octupole contributions to the Sun's field that grow with increasing solar activity. The number of high speed "streams" near the ecliptic plane depends on the number of corrugations of the neutral line that pass through the equator, while the velocities in the near-ecliptic streams depend on both the depths of the corrugations and the tilt of the magnetic dipole axis.

The manner in which high speed streams interact with low speed streams in the solar wind has now been simulated in three dimensions for both tilted planar and warped neutral sheets by Pizzo /19/. Figure 6 shows some cross sections of the structure that can result in the solar wind even for a simple tilted planar dipole neutral line. These models demonstrate that the potential for corotating interplanetary shock formation is greatest at radial distances $\geq 2 \mathrm{AU}$ for conditions when the neutral line is either highly warped for low solar dipole inclinations, or when the solar dipole is moderately inclined. They also show the latitudinal extent of the stream structure for a particular neutral line configuration. Because the details of the neutral line evolution differ from solar cycle to solar cycle $/ 23 /$, the conditions for high shear in the ecliptic must generally be assessed on a case by case basis. For example, conditions during the declining and rising phases of solar activity are often more conducive to strong stream structure than solar minimum conditions. The polar coronal holes are well developed at these times and there are wellseparated neutral sheet crossings of the ecliptic that are accompanied by deep polar coronal hole extensions (see Figure 5). In contrast, at solar minimum the neutral sheet can be quite flat and has low inclination, leading to only weak stream structure near the ecliptic. At solar maximum the large contribution of higher order moments to the solar magnetic field is such that large, well-developed coronal holes do not generally form. Thus solar maximum conditions, which are prime for the transient CME source of interplanetary shocks, are not prime for the stream interaction source. For example, Figure 7 shows the number of stream interaction regions (not shocks) observed during solar cycle 21 at $0.7 \mathrm{AU}$ by Lindsay et al. $/ 5 /$ which suggests how the stream in teraction source varied at larger radial distances. In this case the occurrence rate of stream interaction regions peaked just after the 1986 solar minimum and became comparable to the rate of detected CMEs during the preceding 1979-80 solar maximum. The stream interaction source variation should be more distinctive from cycle to cycle than the CME source variation because of its dependence on the details of the nondipolar solar magnetic fields. 


\section{CONCLUSIONS}

Our understanding of the sources of interplanetary shocks has been significantly enhanced by the availability of observations throughout much of the heliosphere and over long periods of time. These observations, coupled with sophisticated new 3-D models, show us that interplanetary shocks are generally inner-to-middle heliosphere features of the solar wind. The coronal transient or CME source of these shocks exhibits a strong solar activity cycle, while the stream interaction source has a cycle related to but separate from solar activity. The transient source dominates the inner heliosphere at radial distances $\leq 2 \mathrm{AU}$, while stream interactions probably compete with or dominate this source between about 3 and $10 \mathrm{AU}$. Few shocks are found beyond $\sim 20 \mathrm{AU}$. Both sources contribute to shock activity at moderate to high heliolatitudes, with interesting differences from their lower latitude counterparts related to the 3-D properties of stream structure and higher speed of the ambient solar wind. As the Ulysses mission continues, further data from the highest heliolatitudes at both solar minimum and maximum should become available. The WIND and ACE spacecraft will also add to the temporal variability record. In the interim, the context of these forthcoming observations can be better clarified by collective reassessment of the data from earlier missions. As demonstrated here, published results from the different missions cannot readily be intercompared nor can the two sources of interplanetary shocks be generally distinguished. With the newly accessible archives of interplanetary data, one should be able to obtain still better insight from the currently available measurements than is evident from the contents of this review.

\section{ACKNOWLEDGEMENTS}

The author benefitted from discussions with J. Gosling, P. Gazis, J. Mihalov and V. Pizzo. J. Mihalov also provided previously unpublished data for use in Figure 8.

\section{REFERENCES}

1. E. J. Smith, Observations of interplanetary shocks: Recent progress, Space Sci. Rev., 34, 101 (1983).

2. E. J. Smith, Interplanetary shock phenomena beyond $1 \mathrm{AU}$, in: Collisionless Shocks in the Heliospher: Reviews of Current Research, ed. R. G. Stone and B. T. Tsurutani, Geophysical Monograph 35, AGU, Washington, DC 1985, p. 69.

3. J. T. Burkepile and O. C. St. Cyr, A revised and expanded catalogue of mass ejections observed by the solar maximum mission coronagraph, NCAR Tech. Note NCAR TN-369 \& STR, NCAR, Boulder, Colorado (1993).

\section{J. T. Gosling, in Encyclopedia of the Solar System, Academic Press, in press, 1994.}

5. G. M. Lindsay, C. T. Russell, and J. G. Luhmann, On the sources of interplanetary shocks at 0.72 AU, J. Geophys. Res., 99(A1), 11-17 (1994).

6. D. F. Webb and R. A. Howard, The solar cycle variation of coronal mass ejections and the solar wind mass flux, J. Geophys. Res., 99(A3), 4201-4220 (1994).

7. S. Kahler, Coronal mass ejections, Rev. Geophys, , 25, 663 (1987).

8. S. W. Kahler, Solar flares and coronal mass ejections, Ann. Rev. Astron. Astrophys., 30, 113 (1992).

9. J. T. Gosling, The solar flare myth, J. Geophys. Res., 98, 18,937 (1993).

10. C. T. Russell, J. T. Gosling, R. D. Zwickl and E. J. Smith, Multiple spacecraft observations of interplanetary shocks: ISEE three-dimensional plasma measurements, J. Geophys. Res., 88, 9941 (1983).

11. J. T. Gosling and D. J. McComas, Field line draping about fast coronal mass ejecta: A source of strong out-of-ecliptic interplanetary magnetic fields, Geophys. Res. Lett., 14, 355 (1987). 
12. L. F. Burlaga, Magnetic clouds, in: Physics of the Inner Heliosphere II, ed. R. Schwenn and E. Marsch, Springer Verlag, New York 1991, p.1-22.

13. J. T. Gosling, Coronal mass ejections and magnetic flux ropes in interplanetary space, in: Physics of Magnetic Flux Ropes, ed. C. T. Russell, E. R. Priest and L. C. Lee, Geophys. Monograph 58, AGU, Washington, DC 1990, p. 343.

14. J. T. Gosling, D. J. McComas, J. L. Phillips and S. J. Bame, Counterstreaming solar wind halo electron events: Solar cycle variations, J. Geophys. Res., 97, 6531 (1992).

15. H. V. Cane, The large scale structure of flare-associated interplanetary shocks, J. Geophys. Res., 93, 1 (1988).

16. A. J. Hundhausen and R. A. Gentry, Numerical simulation of flare-generated disturbances in the solar wind, J. Geophys. Res., 74(11), 2908-2918 (1969).

17. A. J. Hundhausen, J. T. Burkepile, and O. C. St. Cyr, Speeds of coronal mass ejections: SMM observations from 1980 and 1984-1989, J. Geophys. Res., 99(A4), 6543-6552 (1994).

18. V. Pizzo, A three-dimensional model of corotating streams in the solar wind 3 . Magnetohydrodynamic streams, J. Geophys. Res., 무, 4374 (1982).

19. V. J. Pizzo, Global, quasi-steady dynamics of the distant solar wind. 1. Origin of north-south flows in the outer heliosphere, J. Geophys. Res., 99, 4173 (1994).

20. V. Pizzo, A three dimensional model of corotating streams in the solar wind. 1. Theoretical foundations, J. Geophys. Res. 83, 5563 (1978).

21. X. P. Zhao and A. J. Hundhausen, Organization of solar wind plasma properties in a tilted, heliomagnetic coordinate system, J. Geophys. Res., 흐, 5423 (1981).

22. J. B. Zirker, Coronal holes and high speed wind streams, Rev. Geophys. Space Phys. 15, 257 (1977).

23. T. J. Hoeksema, Large scale configuration of the heliospheric magnetic field: 1976-1991, in: Solar Wind Seven, ed. E. Marsch and R. Schwenn, Pergamon, New York 1992, p. 191.

24. P. M. Volkmer and F. M. Neubauer, Statistical properties of fast magnetoacoustic shock waves in the solar wind between $0.3 \mathrm{AU}$ and $1 \mathrm{AU}$ : Helios-1, 2 observations, Ann. Geophysicae, 3,1 (1985).

25. N. R. Sheeley, Jr., R. A. Howard, M. J. Koomen, D. J. Michels, R. Schwenn, K. H. Mühlhäuser, and H. Rosenbauer, Coronal mass ejections and interplanetary shocks, J. Geophys. Res., 90(A1), 163-175 (1985).

26. J. D. Mihalov, Distant heliospheric results on interplanetary shock propagation, J. Geophys. Res., 90, 209 (1985).

27. P. R. Gazis and A. J. Lazarus, The radial evolution of the solar wind, 1-10 AU, Solar Wind 5, in: NASA Conf. Publ. 2280, ed. M. Neugebauer, NASA, Washington DC 1983, p. 505.

28. L. F. Burlaga, Shocks in the outer heliosphere: Voyager 2 observations from $18.9 \mathrm{AU}$ to $30.2 \mathrm{AU}$ (1986-1989), J. Geophys. Res., 99(A3), 4161-4171 (1994).

29. C. T. Russell, M. M. Hoppe, and W. A. Livesey, Overshoots in planetary bow shocks, Nature, 296, 45 (1982).

30. J. T. Gosling, S. J. Bame, D. J. McComas, J. L. Phillips, V. J. Pizzo, B. E. Goldstein, and M. Neugebauer, Latitudinal variation of solar wind corotating stream interaction regions: Ulysses, Geophys. 
Res. Lett., 20(24), 2789-2792 (1993).

31. V. J. Pizzo, Global, quasi-steady dynamics of the distant solar wind 2. Deformation of the heliospheric current sheet, J. Geophys. Res., 99(A3), 4185-4191 (1994).

32. J. T. Gosling, S. J. Bame, D. J. McComas, J. L. Phillips, E. E. Scime, V. J. Pizzo, B. E. Goldstein, and A. Balogh, A forward-reverse shock pair in the solar wind driven by over-expansion of a coronal mass ejection: Ulysses observations, Geophys. Res. Lett., 21(3), 237-240 (1994).

33. A. K. Richter, K. C. Hsieh, A. H. Luttrell, E. Marsch and R. Schwenn, Review of interplanetary shock phenomena near and within $1 \mathrm{AU}$, in: Collisionless Shocks in the Heliosphere: Reviews of Current Research, ed. R. G. Stone and B. T. Tsurutani, Geophys. Monograph 35, AGU, Washington, DC 1985, p. 33. 